\title{
The timing and deterrence of terrorist attacks due to exogenous dynamics
}

\author{
$\mathrm{K}$ Hausken $^{1 *}$ and $\mathrm{J}$ Zhuang ${ }^{2 *}$ \\ ${ }^{1}$ University of Stavanger, Stavanger, Norway; and ${ }^{2}$ University at Buffalo, State University of New York, \\ Buffalo, NY, USA
}

In this paper, we develop a model for the timing and deterrence of terrorist attacks due to exogenous dynamics. The defender moves first and the attacker second in a two-stage game which is repeated over $T$ periods. We study the effects of dynamics of several critical components of counter-terrorism games, including the unit defence costs (eg, immediately after an attack, the defender would easily acquire defensive funding), unit attack costs (eg, the attacker may accumulate resources as time goes), and the asset valuation (eg, the asset valuation may change over time). We study deterministic dynamics and conduct simulations using random dynamics. We determine the timing of terrorist attacks and how these can be deterred.

Journal of the Operational Research Society (2012) 63, 726-735. doi:10.1057/jors.2011.79

Published online 14 September 2011

Keywords: terrorism; terror capacity; threat; conflict; dynamics; contest success function

\section{Introduction}

Terrorism threats remain substantial. Essential for terrorism assessment is the understanding of the attacker's and defender's objectives, their capacities to attack and defend, and determining how attacks can be deterred as time progresses. We model objectives as utilities, distinguish between unit costs of defence and attack, allow different asset valuations for the defender and attacker, and allow for an inherent defence level. The interplay of these parameters causes a variety of different equilibrium strategies which are analysed.

We develop a model for the timing and deterrence of terrorist attacks in a $T$-period game. The defender builds the defence of infrastructures over time. We assume that the terrorist takes this defence information as given when choosing its attack strategy at each period. Accordingly, in each period, we analyse a two-stage game where the defender moves in the first stage, and the attacker moves in the second stage. Such a game is usually more descriptive than a simultaneous game where the players are unaware of each other's actions.

The two-stage game is played $T$ times referred to as periods. The time between periods is assumed to be sufficiently longer than the time between stages so that

\footnotetext{
${ }^{*}$ Correspondence: $K$ Hausken, Faculty of Social Sciences, University of Stavanger, N-4036 Stavanger, Norway.

E-mail: kjell.hausken@uis.no

$J$ Zhuang, Department of Industrial and Systems Engineering, University at Buffalo, State University of New York, Buffalo, NY 14260, USA. E-mail: jzhuang@buffalo.edu
}

each two-stage game can be solved with backward induction for each period. This means that the players are myopic and boundedly rational in the sense that they only consider one two-stage game in each period. As parameters change through time, the players' strategies change, as we illustrate. Future research may consider how players jointly consider a finite or infinite number of future time periods, which is more complicated to analyse, and may in some cases exceed the players' capabilities.

We consider an asset which may be a single asset, a collection of assets, and even an entire infrastructure which the defender seeks to protect and the attacker seeks to destroy. See Bier et al (2007) for a more explicit focus on what to protect among a collection of locations, where the attacker must choose one location to attack. See Zhuang and Bier (2007) for how a defender strikes a balance between protecting against terrorism and protecting against natural disaster.

For a recent survey of work that examines the strategic dynamics of governments versus terrorists, see Sandler and Siqueira (2009). They survey advances in game-theoretic analyses of terrorism, such as proactive versus defensive countermeasures, the impact of domestic politics, the interaction between political and militant fractions within terrorist groups, and fixed budgets. Further, Brown et al (2006) have focused on interdiction models, attackerdefender models, and related defender-attacker-defender models. They assume a hierarchical structure for decision making. In a defender-attacker-defender model, the defender first invests in protecting the infrastructure, subject to a budget constraint. Then, a resource-constrained attack 
is carried out. Finally, the defender operates the residual system as best possible. They exemplify with border control, the US strategic petroleum reserve, and electric power grids.

Raczynski (2004) simulates the dynamic interactions between terror and anti-terror groups. Feichtinger and Novak (2008) use dynamic game theory to study the intertemporal strategic interactions of Western governments and terror organizations. They illustrate long-run persistent oscillations. Berman and Gavious (2007) study a leader follower game where the State provides counterterrorism support across multiple metropolitan areas to minimize losses, while the terrorist attacks one of the metropolitan areas to maximize his utility. Berrebi and Lakdawalla (2007) consider for 1949-2004 how terrorists seek targets in Israel, responding to costs and benefits, and find that long periods without an attack signal lower risk for most localities, but higher risk for important areas. Barros et al (2006) apply parametric and semi-parametric hazard model specifications to study durations between ETA's terrorist attacks which express seasonal variations with increase in summer and decrease with respect to, for example, deterrence and political variables. Telesca and Lovallo (2006) find temporal correlations in attacks. That is, a terror event is not independent from the time elapsed since the previous event, except for severe attacks which approach a Poisson process. Clauset et al (2007) consider the frequency of severe terrorist events. Udwadia et al (2006) consider the dynamic behaviour of terrorists, those susceptible to terrorist and pacifist propaganda, and pacifists, and military/police intervention to reduce the terrorist population, and nonviolent, persuasive intervention to influence the susceptibles to become pacifists. Hausken and Zhuang (2011a) analyse how a government allocates its resources between attacking in order to downgrade a terrorist's resources, and defending against a terrorist attack. Analogously, the terrorist allocates its resources between attacking a government's asset and defending its own resources. Hausken and Zhuang (2011b) study a two-period game between a government, and a terrorist who decides whether to stockpile attack resources from the first to the second period.

Some research has focused on investment substitutions across time. First, Enders and Sandler (2003) suggest that a terrorist may compile and accumulate resources during times when the defender's investments are high, awaiting times when the defender may relax his efforts and choose lower investments. Second, Keohane and Zeckhauser (2003, pp 201, 224) show that 'the optimal control of terror stocks will rely on both ongoing abatement and periodic cleanup' of 'a terrorist's "stock of terror capacity"'. Enders and Sandler (2005) use time series to show that little has changed in overall terrorism incidents before and after $9 / 11$. Using $9 / 11$ as a break date, they find that logistically complex hostage-taking events have fallen as a proportion of all events, while logistically simple, but deadly, bombings have increased as a proportion of deadly incidents. Bandyopadhyay and Sandler (2011) consider the interaction between preemption and defence. For example, high-cost defenders may rely on preemption, while too little preemption may give rise to subsequent excessive defence.

Hausken et al (2009) consider a defender which chooses tradeoffs between investments in protection against natural disaster only, protection against terrorism only, and all-hazards protection, allowing sequential or simultaneous moves. Similarly, Zhuang and Bier (2007) study the balance between natural disaster and terrorism, where either the defender moves first (and the attacker second), or they move simultaneously. Levitin and Hausken (2008) consider a two-period model where the defender, moving first, distributes its resource between deploying redundant elements and protecting them from attacks. Hausken (2008) considers a terrorist which defends an asset which grows from the first to the second period. The attacker seeks to eliminate the asset optimally across the two periods.

Section 2 develops a model where the defender moves first and the attacker moves second. Section 3 analyses the one-period, two-stage model applying backward induction. Section 4 presents simulations for $T$-period games. In particular, the defender may bounce back after an attack due to a decrease in its unit defence cost. We consider random dynamics of minimal unit defence costs after each attack, random dynamics of unit defence costs in each period, repeated attacks assuming deterministic dynamics of the attacker's unit cost, repeated attacks assuming random dynamics of attacker's unit cost, and repeated attacks due to effects of the dynamics of asset valuation. Section 5 discusses the assumptions and characteristics. Section 6 concludes.

\section{The model}

\subsection{Notation}

$\begin{array}{ll}T & \begin{array}{l}\text { number of time periods } \\ \text { time period, } t=1, \ldots, T\end{array} \\ d_{t} & \begin{array}{l}\text { defender's effort protecting the asset in } \\ \text { period } t, d_{t} \geqslant 0\end{array} \\ A_{t} & \begin{array}{l}\text { attacker's effort in period } t, A_{t} \geqslant 0 \\ \text { inherent defence level in period } t ; \text { for } \\ \text { example, the sunk cost or carried-over }\end{array} \\ c_{t} & \begin{array}{l}\text { defence from history } \\ \text { probability of asset damage in period } t \\ \text { defender's unit cost of effort in period } t\end{array} \\ P_{t}\left(d_{t}, A_{t}\right) & \begin{array}{l}\text { attacker's unit cost of effort in period } t \\ b_{t}\end{array} \\ B_{t} & \begin{array}{l}\text { defender's valuation of the asset } \\ v_{t}\end{array} \\ V_{t} & \begin{array}{l}\text { attacker's valuation of the asset } \\ \text { defender's expected utility in period } t\end{array}\end{array}$


$U_{t}\left(d_{t}, A_{t}\right)$

$A_{t}\left(d_{t}\right)$

attacker's expected utility in period $t$ attacker's best response function; that is,

$$
A_{t}\left(d_{t}\right) \equiv \arg \max _{A_{t} \geqslant 0} U_{t}\left(d_{t}, A_{t}\right)
$$

\subsection{Assumptions}

For the probability of damage, we consider the common ratio form (Tullock, 1980; Skaperdas, 1996; Amegashie, 2006; Zhuang and Bier, 2007, pp 981, 983) contest success function,

$$
P_{t}\left(d_{t}, A_{t}\right)=\frac{A_{t}}{A_{t}+d_{t}+c_{t}}
$$

where $\partial P_{t} / \partial A_{t}>0$ and $\partial P_{t} / \partial d_{t}<0$. We have included $c_{t}$ which is the inherent defence level at the beginning of period $t$, which may depend on the histories of the players' strategies $\left\{d_{t-1}, d_{t-2}, \ldots\right\}$ and $\left\{A_{t-1}, A_{t-2}, \ldots\right\}$, and damage $\left\{P_{t-1}, P_{t-2}, \ldots\right\}$.

The defender and attacker value the asset at $v_{t}$ and $V_{t}$, respectively, simplistically referred to as the asset valuations. The probability that the asset is not damaged is $1-P_{t}\left(d_{t}, A_{t}\right)$, which the defender maximizes, accounting for the valuation $v_{t}$ and defence expenditures $b_{t} d_{t}$. Analogously, the attacker maximizes the damage accounting for the valuation $V_{t}$, and attack expenditures $B_{t} A_{t}$. The defender's and attacker's expected utilities in period $t$ are

$$
\begin{aligned}
u_{t}\left(d_{t}, A_{t}\right) & =\left[1-P_{t}\left(d_{t}, A_{t}\right)\right] v_{t}-b_{t} d_{t} \\
& =\frac{d_{t}+c_{t}}{A_{t}+d_{t}+c_{t}} v_{t}-b_{t} d_{t} \\
U_{t}\left(d_{t}, A_{t}\right) & =P_{t}\left(d_{t}, A_{t}\right) V_{t}-B_{t} A_{t} \\
& =\frac{A_{t}}{A_{t}+d_{t}+c_{t}} V_{t}-B_{t} A_{t}
\end{aligned}
$$

Each actor has a benefit term with $v_{t}$ or $V_{t}$, and a cost term with $d_{t}$ or $A_{t}$. Since each actor weighs benefits against costs, we do not impose constraints on $d_{t}$ and $A_{t}$. There may be cases where an actor has scarce resources. For such cases we assume that rational actors are somehow able, for example, through taking up loans, to furnish the resources needed for strategic determination of $d_{t}$ and $A_{t}$. We assume common knowledge so that both players know all parameters and the game structure.

\subsection{Problem formulation}

To determine the Subgame Perfect Nash Equilibrium we assume that the defender chooses $d_{t}$ in the first stage. The attacker observes $d_{t}$ and chooses $A_{t}$ in the second stage. The game is solved with backward induction.
Definition 1 A strategy pair, $\left(d_{t}^{S}, A_{t}^{S}\right)$ is a Subgame Perfect Nash Equilibrium if and only if

$$
A_{t}^{S}=A_{t}\left(d_{t}^{S}\right)=\arg \max _{A_{t} \geqslant 0} U_{t}\left(d_{t}^{S}, A_{t}\right)
$$

and

$$
d_{t}^{S}=\arg \max _{d_{t} \geqslant 0} u_{t}\left(d_{t}, A_{t}\left(d_{t}\right)\right)
$$

\section{Solving the two-stage game}

For any given defence level $d_{t}$, maximizing the attacker's utility $U_{t}\left(d_{t}, A_{t}\right)$ specified in (2) gives the attacker bestresponse function.

$$
\begin{aligned}
A_{t}\left(d_{t}\right)= & \arg \max _{A_{t} \geqslant 0} U_{t}\left(d_{t}, A_{t}\right) \\
& = \begin{cases}0 & \text { if } V_{t} \leqslant B_{t} c_{t} \\
0 & \text { if } V_{t}>B_{t} c_{t}, \text { and } \\
\sqrt{\frac{V_{t}}{B_{t}}\left(d_{t}+c_{t}\right)}-d_{t}-c_{t} & \text { otherwise }\end{cases}
\end{aligned}
$$

where $\bar{d}_{t}$ is the minimum level of defensive investment required to deter the attacker altogether. Observe that $\bar{d}_{t}$ increases in the attacker's valuation $V_{t}$, increases if the attacker's unit attack cost $B_{t}$ decreases, and the defender's inherent defence level $c_{t}$ is subtracted. If $\bar{d}_{t}<0$, which implies $V_{t} \leqslant B_{t} c_{t}$, the inherent defence level itself deters the attacker. This occurs when the attacker's valuation divided by his unit attack cost is lower than the inherent defence level as shown in the first line in (5). This implies neither defence nor attack. The second line in (5) expresses that if $V_{t}>B_{t} c_{t}$, then the attacker will be deterred in the second stage by the defender's first-stage defence larger than a threshold $\bar{d}_{t}$. This implies defence but no attack. The third line in (5) expresses the interior solution where the defender's first-stage defence is moderately high (not deterrent), after which the attacker chooses a positive attack in the second stage.

Substituting the attacker's best response $A_{t}\left(d_{t}\right)$ in (5) into the defender's optimization problem (4), Table 1 shows the solution for the Subgame Perfect Nash Equilibrium.

There are four exhaustive and mutually exclusive solution forms. Cases 2 and 3 are not possible if the right-hand side of the respective inequality is less than the left-hand side.

(1) When the attacker's asset valuation is small compared to the difficulty of attack and inherent defence level, $V_{t} \leqslant B_{t} c_{t}$, the attacker does not attack. So we have zero attacker effort and zero defence level at equilibrium.

(2) When the attacker's valuation is large compared with the difficulty of attack and inherent defence level, 
Table 1 Solution to Subgame Perfect Nash equilibrium

\begin{tabular}{lllllll}
\hline Cases & Conditions & $d_{t}^{S}$ & $A_{t}^{S}$ & $u_{t}^{S}$ & $U_{t}^{S}$ & Scenarios \\
\hline 1 & $V_{t} \leqslant B_{t} c_{t}$ & 0 & 0 & $v_{t}$ & No defence/attack \\
2 & $B_{t} c_{t} \leqslant V_{t} \leqslant \frac{B_{t} v_{t}}{2 b_{t}}$ & $\frac{V_{t}}{B_{t}}-c_{t}$ & 0 & $v_{t}-b_{t}\left(\frac{V_{t}}{B_{t}}-c_{t}\right)$ & 0 & Attacker deterred \\
3 & $B_{t} \operatorname{Max}\left\{c_{t}, \frac{v_{t}}{2 b_{t}}\right\} \leqslant V_{t} \leqslant \frac{B_{t} v_{t}^{2}}{4 c_{t} b_{t}^{2}}$ & $\frac{B_{t} v_{t}^{2}}{4 b_{t}^{2} V_{t}}-c_{t}$ & $\frac{v_{t}}{2 b_{t}}-\frac{B_{t} v_{t}^{2}}{4 b_{t}^{2} V_{t}}$ & $\frac{B_{t} v_{t}^{2}}{4 b_{t} V_{t}}+b_{t} c_{t}$ & $V_{t}-\frac{B_{t} v_{t}}{b_{t}}+\frac{B_{t}^{2} v_{t}^{2}}{4 b_{t}^{2} V_{t}}$ & Defence/attack \\
4 & $V_{t} \geqslant B_{t} \operatorname{Max}\left\{c_{t}, \frac{v_{t}}{2 b_{t}}, \frac{v_{t}^{2}}{4 c_{t} b_{t}^{2}}\right\}$ & 0 & $\sqrt{\frac{V_{t} c_{t}}{B_{t}}-c_{t}}$ & $\sqrt{\frac{B_{t} c_{t}}{V_{t}} v_{t}}$ & $\left(\sqrt{V_{t}}-\sqrt{B_{t} c_{t}}\right)^{2}$ & No defence \\
\hline
\end{tabular}

$V_{t} \geqslant B_{t} c_{t}$, but the defender's valuation is at least twice (weighted by cost units $B_{t}$ and $b_{t}$ ) larger than the attacker's, $V_{t} \leqslant\left(B_{t} v_{t}\right) /\left(2 b_{t}\right)$, the defence level is positive, and the attacker is deterred by this positive defence level. This means that the attacker can be deterred with a positive defence level at some Subgame Perfect Nash equilibrium; that is, there exists some Subgame Perfect Nash equilibrium $\left(d_{t}^{S}, A_{t}^{S}\right)$ such that $A_{t}^{S}=0$ and $d_{t}^{S}>0$.

(3) When the attacker's valuation is larger than in cases 1 and 2, expressed with $B_{t} \operatorname{Max}\left\{c_{t},\left(v_{t}\right) /\left(2 b_{t}\right)\right\} \leqslant V_{t}$, but not very large $V_{t} \leqslant\left(B_{t} v_{t}^{2}\right) /\left(4 c_{t} b_{t}^{2}\right)$, both the defence and attack levels are positive. The attacker attacks despite meeting resistance.

(4) When the attacker's valuation is very large, that is, larger than in cases 1, 2, and 3, the defence level is zero, which gives a positive attack. The corresponding condition is $V_{t} \geqslant B_{t} \operatorname{Max}\left\{c_{t},\left(v_{t}\right) /\left(2 b_{t}\right),\left(v_{t}^{2}\right) /\left(4 c_{t} b_{t}^{2}\right)\right\}$ in order for the four conditions to be collectively exhaustive and mutually exclusive. The three terms within the Max sign are simply the right-hand sides of the inequalities for cases 1, 2, 3. Case 4 is less common and means that the defender is so inferior and overwhelmed that it removes its defence.

As the attacker's valuation increases from case 1 to case 4 in Table 1 , the attacker gradually acquires a more superior position. When the unit attack cost $B_{t}$ and/or the defence level $c_{t}$ are zero, case 1 is impossible; and when $B_{t}$ and/or $c_{t}$ are infinity, case 1 is guaranteed. A high $B_{t} / b_{t}$ specifies a disadvantaged attacker in terms of unit costs, and a high $B_{t} v_{t} / 2 b_{t}$ additionally specifies high defender valuation $v_{t}$, which deters the attacker. A low $c_{t}$ causing a high $B_{t} v_{t}^{2} / 4 c_{t} b_{t}^{2}$ induces attack and defence if $V_{t}$ is below, and if $V_{t}$ is above the defender withdraws from the overwhelming attack.

Example Assuming $V_{t}=50, v_{t}=50, c_{t}=1$, and $b_{t}=1$ imply that $B_{t}=51$ gives $d_{t}^{S}=A_{t}^{S}=0$ (case 1$), B_{t}=2$ gives $d_{t}^{S}=24$ and $A_{t}^{S}=0$ (case 2$), B_{t}=1$ gives $d_{t}^{\mathrm{S}}=11.5$ and
$A_{t}^{S}=12.5$ (case 3), $B_{t}=0.07$ gives $d_{t}^{S}=0$ and $A_{t}^{S}=25.7$ (case 4). Hence, first, when the unit attack costs are extremely high (above 50), no attack occurs and therefore no defence is needed (the inherent defence level provides sufficient defence). Second, when the unit attack costs are high, attacks can be deterred by positive defence, while when the unit attack costs are low, attacks cannot be deterred. Finally, when the unit attack costs are extremely low (below 0.08), the defender gives up.

\section{The $T$-period game}

\subsection{The defender bounces back after an attack}

We assume that the time between periods is sufficiently longer than the time between stages so that each two-stage game can be solved with backward induction. This means that the players are myopic and boundedly rational and only consider one two-stage game in each time period. They thus maximize $u_{t}$ and $U_{t}$, respectively, in each period. Assume that $B_{s} \operatorname{Max}\left\{c_{s}, v_{s} / 2 b_{s}\right\} \leqslant V_{s}$ so that an attack occurs in period $s$. Although several parameters may change after an attack, we consider the defence unit cost $b_{t}$ as the most likely to change substantially. Hence assume for simplicity that $B_{t}=B_{s}, c_{t}=c_{s}$, and $v_{t}=v_{s}$ remain fixed for all $t \geqslant s$, while the defence unit cost $b_{t}$ changes as a result of the attack. This is because after an attack in period $s$ the defender would more easily acquire defensive funding from various sources which, together with political will, decrease the unit defence cost $b_{t}$. With a significantly lower $b_{t}$, the condition $B_{t} \operatorname{Max}\left\{c_{t}, v_{t} / 2 b_{t}\right\} \leqslant V_{t}$ is no longer satisfied. Assume that $b_{t}$ follows the form for $t \geqslant s$ :

$$
b_{t}= \begin{cases}b_{s} & \text { if } t=s \\ b_{s}-\left[b_{s}-b_{\min }\right] \mathrm{e}^{-\Phi(t-s-1)} & \text { if } s<t \leqslant t^{*}\end{cases}
$$

where $b_{\text {min }}$ is the minimum value that $b_{t}$ acquires after an attack $A_{s}$ in period $s$, and $\Phi>0$ regulates how quickly $b_{t}$ bounces back to its original level $b_{s}$ due to defence reinforcement. Since $\operatorname{Lim}_{t \rightarrow \infty} b_{t}=b_{s}$ and $\partial b_{t} / \partial t>0$, a second 
attack eventually occurs in period $t^{*}$ determined by the smallest $t=t^{*}$ such that $B_{t} \operatorname{Max}\left\{c_{t}, v_{t} / 2 b_{t}\right\} \leqslant V_{t}$ (see case 3 in Table 1) which, when $c_{t}<v_{t} / 2 b_{t}$, implies

$$
t^{*}=\left\lceil s+1+\frac{1}{\Phi} \operatorname{Ln}\left(\frac{b_{s}-b_{\min }}{b_{s}-B_{t} v_{t} / 2 V_{t}}\right)\right\rceil
$$

where $\lceil z\rceil$ is the least integer that is not less than $z$. When $\Phi=0, b_{t}=b_{\min }\left(A_{s}\right)$ for all $t>s$ and the attacker is always deterred. When $\Phi=\infty, b_{t}=b_{s}$ for all $t>s$ and the attacker attacks in each subsequent period. Figure 1 shows the five equilibrium values $u_{t}, U_{t}, d_{t}, A_{t}$, and $b_{t}$ as functions of time $t$ when $V_{t}=v_{t}=2, B_{t}=c_{t}=1, b_{s}=0.6, s=0$, $b_{\min }=0.3$, and $\Phi=0.25$.

Figure 1 shows that due to the low defence cost, the defence level is high at 1 to fully deter the attacks for the first five periods after the original attack, which corresponds to case 2 of Table 1 . The defender's unit defence cost $b_{t}$ increases gradually through the six periods according to (6), which causes the defender's utility to decrease. The next attack happens in period $t^{*}=6$, when the unit defence cost grows to $b_{t^{*}}=0.514>0.5$ such that the inequality $B_{t^{*}} \operatorname{Max}\left\{c_{t^{*}}, \quad v_{t^{*}} / 2 b_{t^{*}}\right\}=1.945 \leqslant 2=V_{t^{*}}$ no longer holds. The defender's defence $d_{t}$ stays at 1 for the first five periods, and drops to 0.89 in period 6 reflecting that when the defence is sufficiently expensive, the defender, moving first in period 6, accepts some asset damage rather than protecting the asset more thoroughly. In the first five periods the attacker refrains from attack earning zero utility, and attacks in period 6 earning a positive utility.

\subsection{Random dynamics of minimal unit defence costs after each attack}

Assume that once an attack happens in period $t, b_{\min }$ is determined by $\operatorname{Max}\left[0, N\left(0.5 b_{t}, 0.5 b_{t}\right)\right]$ in period $t+1$, that is, a normal random variable with a mean and standard deviation both equalling $0.5 b_{t}$. The motivation for this randomness is that $b_{\min }$ in (6) and (7) is determined by a political process which can be quite unpredictable, but may well fluctuate as in a Gaussian distribution. Then the unit defence cost and next attack time will be determined by (6) and (7), respectively. Figure 2 shows this dynamics. Periods 1-6 are equivalent in Figures 1 and 2. After the attack in period 6, the defender unit cost drops to 0.19 in period 7 , drawn from the random distribution. Thereafter no attack occurs and no random draws are made in periods $7-12$. In period 13, an attack happens because the defence is no longer cheap $\left(b_{t}>0.5\right)$. In period 14 , the minimal defence cost drops to 0.42 due to the random draw, which deters attacks in only three periods (periods 14-16). Observe how $b_{\text {min }}$ fluctuates through time, reflecting different defender reactions after each attack.

\subsection{Random dynamics of unit defence costs in each period}

In this section, we assume the unit defence cost $b_{t}$ is randomly given by $\operatorname{Max}\left[N\left(b_{t}^{\prime}, 0.2 b_{t}^{\prime}\right), 0\right]$ in each time period; that is, the maximum of 0 and a normal random number with mean $b_{t}^{\prime}$ and standard deviation $0.2 b_{t}^{\prime} . b_{t}^{\prime}$ is determined by (6) setting $s=t$ when an attack occurs, where $b_{t}^{\prime}$ can drop as a result of an attack before a new random draw of $b_{t}$. We use the same parameter values as in

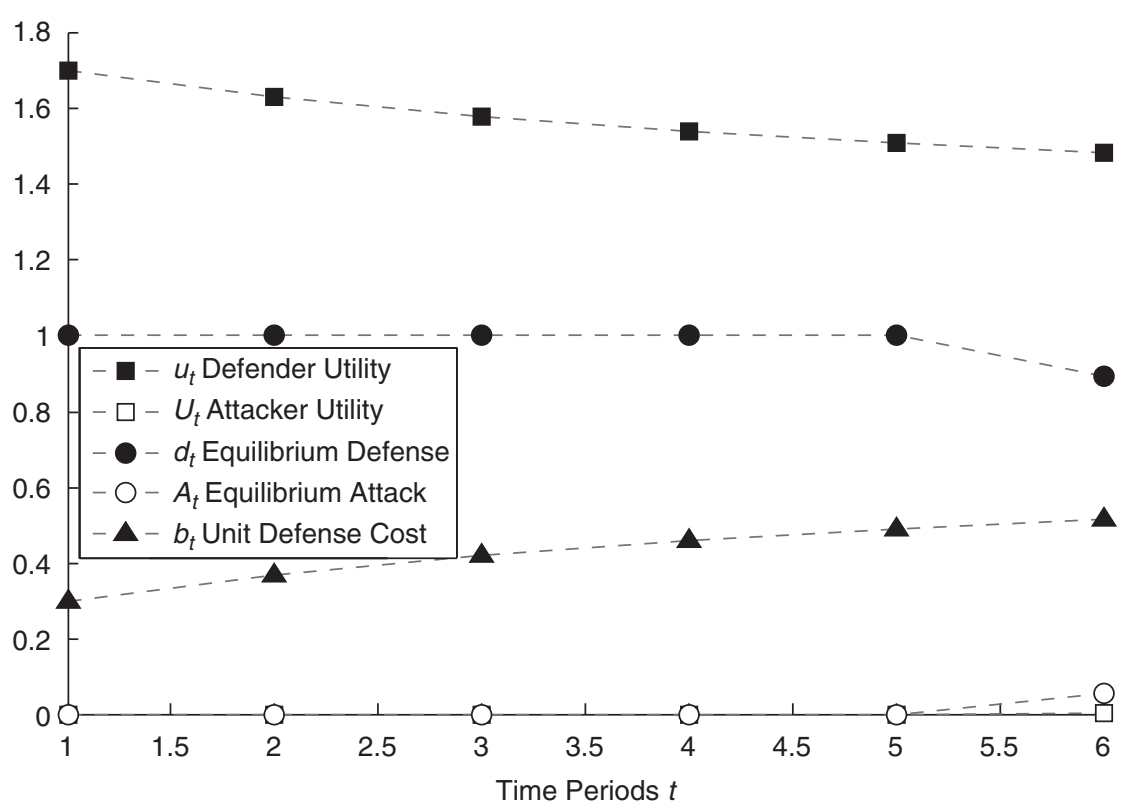

Figure 1 Relationship between equilibrium and unit defence costs of deterministic dynamics. 


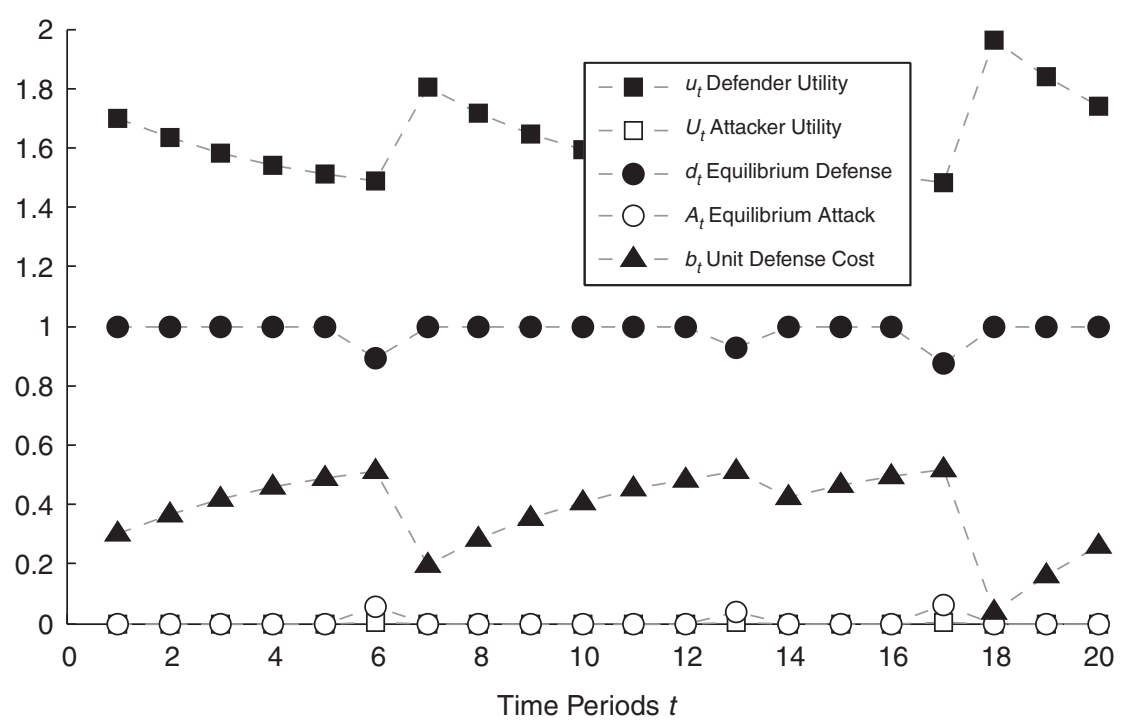

Figure 2 Repeated attacks due to the random dynamics of minimal unit defence costs.

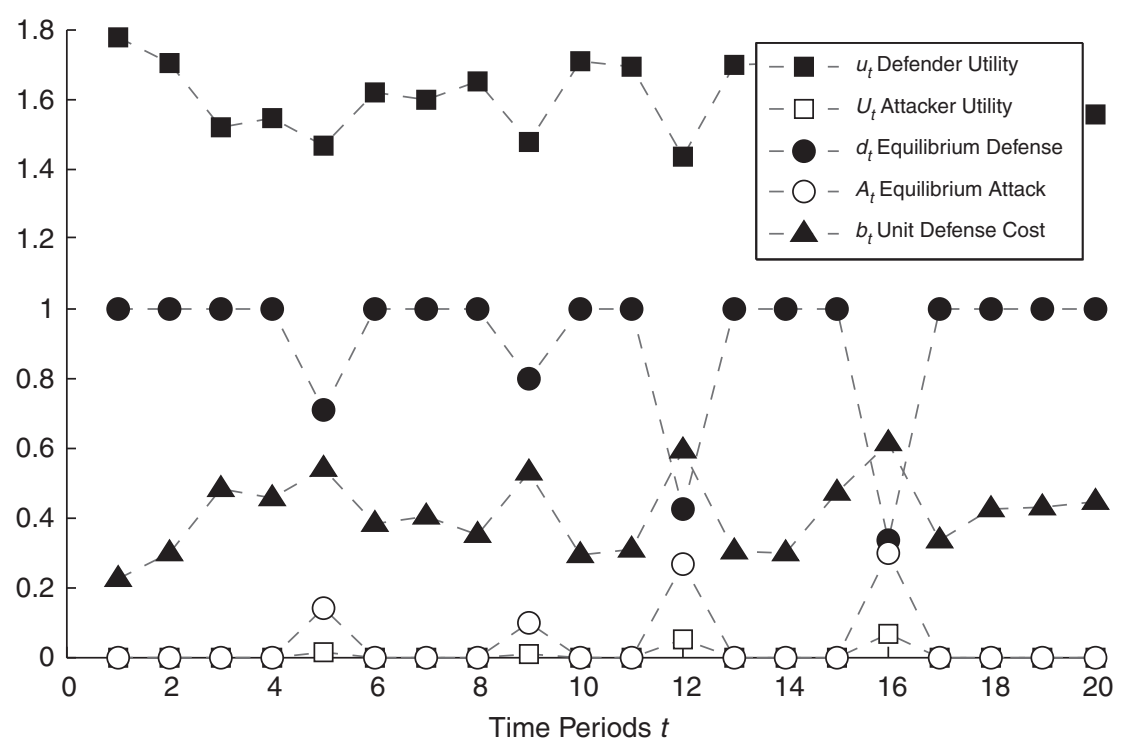

Figure 3 Repeated attacks due to the random dynamics of unit defence costs.

Section 4.1. Starting with $b_{0}=0.6$, an attack occurs when $t=0$ causing $b_{1}^{\prime}=b_{\min }=0.3$.

Thus $b_{1}$ follows from drawing from $\operatorname{Max}[N(0.3$, $0.2 \times 0.3), 0]$. Equation (6) is used to determine $b_{2}^{\prime}$ and $b_{2}$ is determined by a new draw, and analogously for periods 3, 4, etc. Figure 3 shows one simulation result. In period 5 the unit defence cost reaches a high value $b_{t}=0.54>0.5$ and as a result the second attack happens. In period 6 , the unit defence cost $b_{6}$ is drawn from $\operatorname{Max}[N(0.3,0.2 \times 0.3), 0]$, following the same distribution as $b_{1}$; and so on and so forth. In period $9, b_{t}=0.53$, causing a third attack. The fourth and fifth attacks happen in periods 12 and 16 , respectively.
4.4. Repeated attacks assuming deterministic dynamics of attacker's unit cost $B_{t}$

We consider $T=100$ and we use the following formula to determine the unit attack cost $B_{t}$ :

$$
B_{t}= \begin{cases}10 & \text { if } t=1 \\ 0.95 B_{t-1} & \text { if } t>1, \text { and } A_{t-1}=0 \\ 10 & \text { if } t>1, \text { and } A_{t-1}>0\end{cases}
$$

That is, we start with a unit attack cost of 10 . If the attacker effort is zero in the previous period, we assume the attacker accumulates more resources over time and therefore the unit cost decreases by $5 \%$. Otherwise, if the 


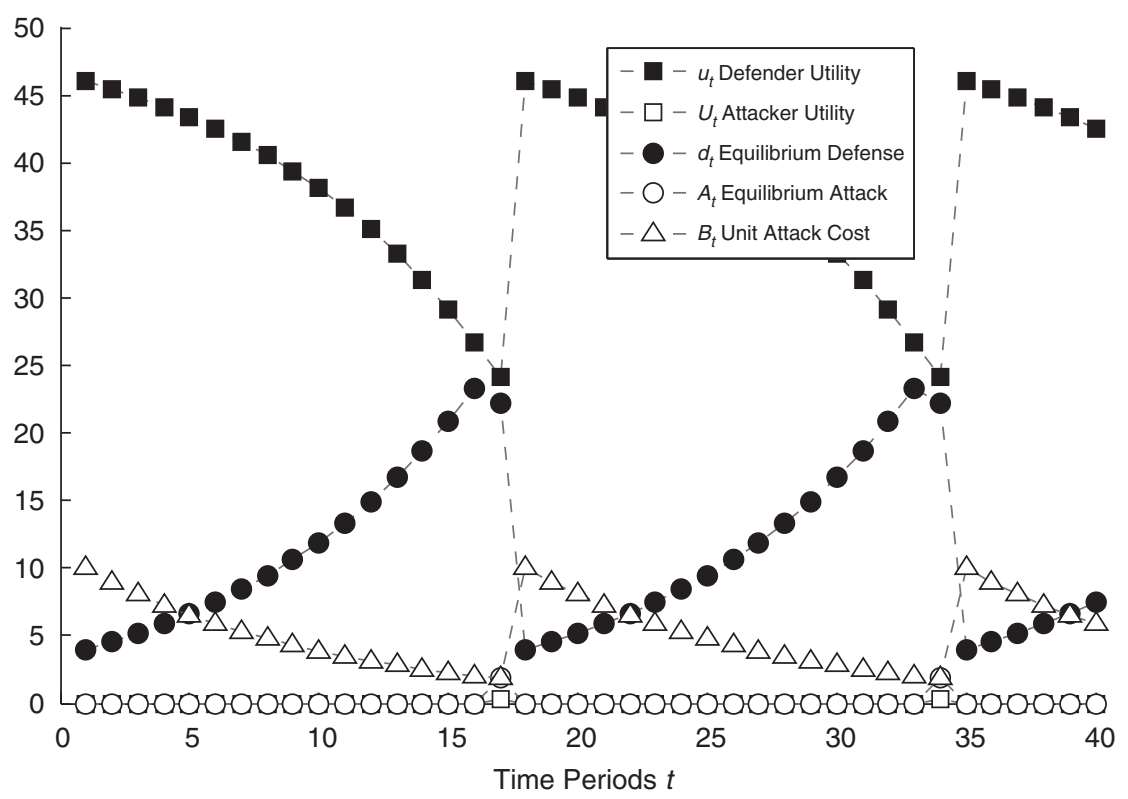

Figure 4 Relationship between equilibrium attacker efforts and unit attack costs of deterministic dynamics.

attacker effort is positive in the previous period, $B_{t}$ returns to the initial level 10. Figure 4 illustrates when $V_{t}=50$ and $v_{t}=50, c_{t}=1, b_{t}=1$ for all $t=1, \ldots, T$.

From Figure 4, we see the following: first, the defence level is always positive because of the high defender asset valuation. Second, when the unit attack cost decreases, the defender has to increase its defence to keep deterring the possible attacks. Third, once the unit attack cost drops below a threshold such that $V_{t} \geqslant B_{t} v_{t} / 2 b_{t}$, an attack happens. The deterministic dynamics in (8) is such that the unit attack cost decreases gradually, and as a result a modest attack happens once the unit attack cost reaches a threshold. This is consistent with practical observations where attacks are often modest compared with the vast amounts of resources allocated for example to military defence budgets. Finally, after an attack happens, the unit attack cost returns to a high level and a new cycle happens. In this section we assume no dynamics for $b_{t}$, and hence a low $d_{t}$ occurs after an attack and is sufficient since $B_{t}$ is so large.

\subsection{Repeated attacks assuming random dynamics of attacker's unit cost $B_{t}$}

This section includes a random error term expressed with the normal distribution $N(0,1)$. We consider $T=100$ and we use the following formula to determine the unit attack $\operatorname{cost} B_{t}$ :

$$
B_{t}= \begin{cases}10 & \text { if } t=1 \\ \operatorname{Max}\left\{0,0.95 B_{t-1}+N(0,1)\right\} & \text { if } t>1, \text { and } A_{t-1}=0 \\ \operatorname{Max}\{0,10+N(0,1)\} & \text { if } t>1, \text { and } A_{t-1}>0\end{cases}
$$

where the Max function prevents negative $B_{t}$. The unit attack cost can fluctuate stochastically as in (9) due to many reasons, such as random influx of monetary support, or random freezing of terrorist assets. Using the same parameter values as in Section 4.4, see Figure 5 for illustration. Comparing Figures 4 and 5, we see how the randomness in Figure 5 changes the periodicity of attacks, as well as the levels of attacks and defences. Now substantial attacks are possible due to some sudden drop of the unit attack cost. This reflects many real-world scenarios which have occurred and may occur in the future.

\subsection{Repeated attacks due to effects of the dynamics of asset valuation}

Assume that the attacker's asset valuation decreases as a result of the success of a previous attack, while the defender's target valuation remains the same, as expressed with

$$
V_{t}=V_{t-1} \mathrm{e}^{-P_{t-1}\left(d_{t-1}, A_{t-1}\right)}
$$

The motivation for this assumption is that attacking the same object over and over again, regardless of whether the object is an asset, an infrastructure, or an opponent, becomes less interesting if the object is successfully attacked each time. Then using the same parameter values as in Section 4.4, we get the results in Figure 6.

Figure 6 tells us that the attacker's valuation decreases to around 25 after the attacks in the first two periods. Thereafter the attacker becomes less interested in the asset, and is fully deterred by the defence $d_{t}=24$. 


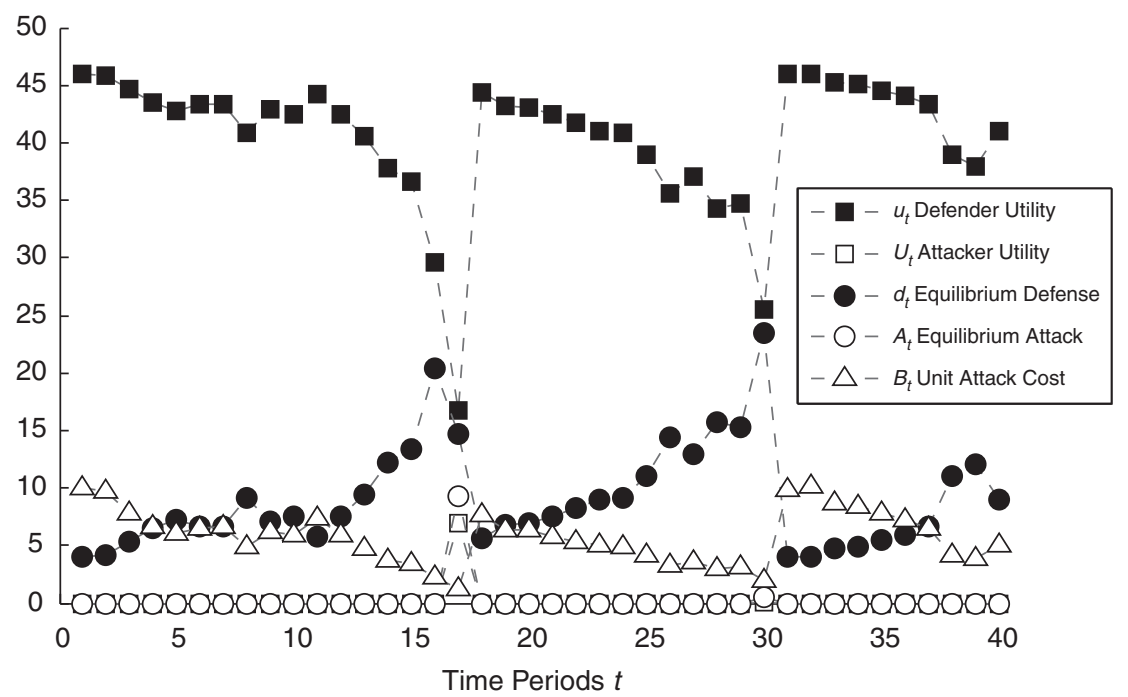

Figure 5 Relationship between equilibrium attacker efforts and unit attack costs of random dynamics.

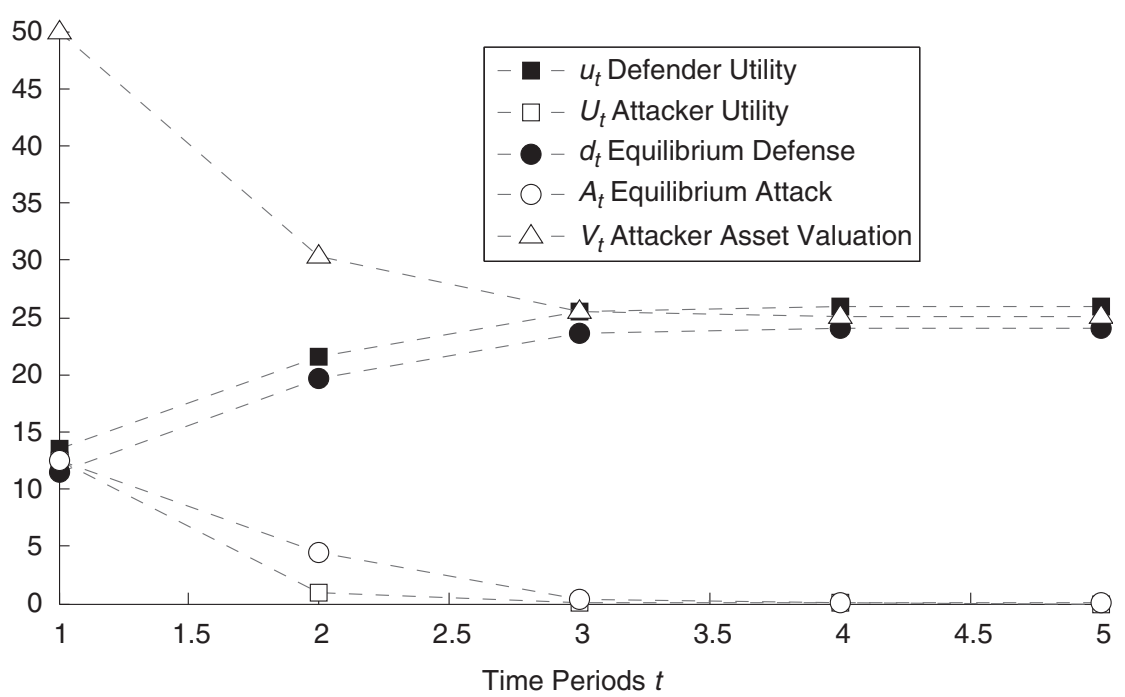

Figure 6 Relationship between equilibrium and the dynamics of attacker asset valuation.

\section{Discussion}

The key feature of this paper is to study that the terrorists can accumulate assets at some rates. In practice, the measurable factors determining the speed to accumulate assets may include: size, structure and organization of terrorist organization, competence within the terrorist organization, popularity among the supporters, current asset level, historical terrorism activities, factors of competing terrorism organizations, and the ability of the government to impact the terrorist's asset accumulation.

In this paper we assume that the attacker costs are linear in the attack effort (ie, marginal attack cost is a constant). This is a reasonable assumption when the attack effort is in the normal range. In practice, when the attack effort is large, the marginal attack costs may increase in the attack effort (eg, running out of willing suicide bombers). Taking this into account, we expect that at equilibrium, the terrorist's utility and attack effort would be weakly smaller, and the defender's utility and defence would be weakly larger.

As mentioned in Section 2, the inherent defence could be either sunk cost (such as personnel expense, which cannot be carried over to the next period), or carried-over defence (eg, infrastructure building) which could depend on the histories of the players' strategies and damage. For simplicity, this paper focuses on the sunk cost but does not study the carried-over case, which has been modelled in Zhuang et al (2010).

As mentioned in Section 3, when the attacker's valuation is very large, the case 4 of the solution may happen, where the defender removes its defence. This case arises when the 
defender's contest success against a superior terrorist is so low that the defence cost is not justified. Military history is replete with examples where defenders flee from superior attackers or invaders. For example, one defending warship does not stand much chance against 100 invading warships. In most terrorism cases to date terrorists have not been that superior, and terrorists have usually not attacked inferior defenders. That is, the terrorist's valuation is usually not sufficiently large compared to the defender's target valuation. However, case 4 may well become more likely in the future and we keep it for theoretical completeness.

\section{Conclusion}

We develop a model for the timing and deterrence of terrorist attacks in a $T$-period game due to exogenous dynamics. The defender controls an asset which is under repeated attacks by a terrorist. The defender's objective is to deter attacks. The agents have different unit costs of defence and attack, different asset valuations, and we allow for an inherent defence level. A defender usually builds infrastructures over time. The defender thus moves first and the attacker second in a two-stage game. This gives four cases which are analysed analytically, that is, neither defence nor attack (if the attacker's valuation is low), attacker deterred, joint defence and attack, and no defence (if the defender is inferior). We analyse how the four cases depend on the parameters. We study deterministic dynamics and conduct simulation using random dynamics. We determine the timing of terrorist attacks.

Repeating the two-stage game, first we allow the defender to bounce back after an attack, caused by a drop in the unit defence cost to a minimum with subsequent concave increase to its initial level before the attack. Second, we let the minimum unit defence cost, after an attack, be drawn from a Gaussian distribution, before subsequent increase, and consider repeated attacks through time. Third, we let the unit defence cost be drawn randomly in each period, with drop to a minimum after each attack. Fourth, we let the unit attack cost be initially high, after which it decreases gradually, for example, caused by incoming funding. This causes the defence to increase to ensure deterrence, but an attack eventually occurs as the attacker grows resourceful. The attack drains resources which increases the unit attack cost, and enables the defender to re-establish its deterrence. Fifth, we allow the unit attack cost to decrease stochastically which allows more substantial attacks. Finally, we let the attacker's asset valuation decrease as a result of previous successful attacks, which makes deterrence easier.

Acknowledgements - This research was partially supported by the United States Department of Homeland Security through the National Center for Risk and Economic Analysis of Terrorism Events
(CREATE) under award number 2010-ST-061-RE0001. However, any opinions, findings, and conclusions or recommendations in this document are those of the authors and do not necessarily reflect views of the United States Department of Homeland Security, or CREATE. Author names were listed alphabetically by last name.

\section{References}

Amegashie JA (2006). A contest success function with a tractable noise parameter. Pub Choice 126: 135-144.

Bandyopadhyay S and Sandler T (2011). The interplay between preemptive and defensive counterterrorism measures: A twostage game. Economica 78(311): 546-564.

Barros CP, Passos J and Gil-Alana LA (2006). The timing of ETA terrorist attacks. J Pol Model 28(3): 335-346.

Berman O and Gavious A (2007). Location of terror response facilities: A game between state and terrorist. Eur J Opl Res 177(2): 1113-1133.

Berrebi C and Lakdawalla D (2007). How does terrorism risk vary across space and time? An analysis based on the Israeli experience. Defence Peace Econ 18(2): 113-131.

Bier VM, Oliveros S and Samuelson L (2007). Choosing what to protect: Strategic defensive allocation against an unknown attacker. J Pub Econ Theory 9: 563-587.

Brown G, Carlyle M, Salmeron J and Wood K (2006). Defending critical infrastructure. Interfaces 36: 530-544.

Clauset A, Young M and Gleditsch KS (2007). On the frequency of severe terrorist events. $J$ Conflict Resolut 51: 58-87.

Enders W and Sandler T (2003). What do we know about the substitution effect in transnational terrorism? In: Silke A and Ilardi G (eds). Research on Terrorism: Trends, Achievements and Failures. Frank Cass: London.

Enders W and Sandler T (2005). After 9/11: Is it all different now? $J$ Conflict Resolut 49(2): 259-277.

Feichtinger G and Novak A (2008). Terror and counterterror operations: Differential game with cyclical Nash solution. J Optim Theory Appl 139(3): 541-556.

Hausken K (2008). Whether to attack a terrorist's resource stock today or tomorrow. Games Econ Behav 64(2): 548-564.

Hausken K, Bier V and Zhuang J (2009). Defending against terrorism, natural disaster, and all hazards. In: Bier VM and Azaiez MN (eds). Game Theoretic Risk Analysis of Security Threats. Springer: New York, pp 65-97.

Hausken K and Zhuang J (2011a). Governments' and terrorists' defense and attack in a T-period game. Decis Anal 8(1): 46-70.

Hausken K and Zhuang J (2011b). Defending against a terrorist who accumulates resources. Military Opns Res 16(1): 21-39.

Keohane N and Zeckhauser RJ (2003). The ecology of terror defense. J Risk Uncertainty 26: 201-229.

Levitin G and Hausken K (2008). Protection vs. redundancy in homogeneous parallel systems. Reliab Eng Syst Saf 93(10): $1444-1451$.

Raczynski S (2004). Simulation of the dynamic interactions between terror and anti-terror organizational structures. J Artif Soc Social Simul 7(2), http://jasss.soc.surrey.ac.uk/7/2/8.html.

Sandler T and Siqueira K (2009). Games and terrorism. Simulation \& Gaming 40(2): 164-192.

Skaperdas S (1996). Contest success functions. Econ Theory 7: 283-290.

Telesca L and Lovallo M (2006). Are global terrorist attacks timecorrelated? Phys A-Statist Mech Appl 362(2): 480-484.

Tullock G (1980). Efficient rent-seeking. In: Buchanan JM, Tollison RD and Tullock G (eds). Toward a Theory of the Rent-Seeking 
Society. Texas A\&M University Press: College Station, pp 97-112.

Udwadia FE, Leitmann G and Lambertini L (2006). A dynamical model of terrorism. Disc Dynam Nature Soc. doi: 10.1155/ DDNS/2006/85653, pp. 1-32.

Zhuang $\mathbf{J}$ and Bier VM (2007). Balancing terrorism and natural disasters-Defensive strategy with endogenous attack effort. Opns Res 55(5): 976-991.
Zhuang J, Bier VM and Alagoz O (2010). Modeling secrecy and deception in a multiple-period attacker-defender signaling game. Eur J Opl Res 203(2): 409-4180.

Received March 2010; accepted March 2011 after one revision 